\title{
Aspectos nutricionais em obesos antes e após a cirurgia bariátrica
}

\section{Nutrition aspects in obese before and after bariatric surgery}

\author{
Isabella Valois Pedrosa; Maria Goretti Pessoa de Araújo Burgos²; Niedja Cristina Souza³; Caroline Neves de Morais ${ }^{4}$
}

\section{RE S U M O}

\begin{abstract}
Objetivo: Determinar perfil clínico-nutricional de pacientes obesos submetidos à cirurgia bariátrica, no HC/UFPE. Métodos: Foram avaliados retrospectivamente, 205 pacientes, no período 2002/2006. A análise considerou história clínica para diabetes tipo 2 (DM 2), hipertensão arterial (HA) e síndrome metabólica (SM). O estado nutricional pré-operatório foi avaliado pelo IMC e bioquímica (hemoglobina, hematócrito, albumina, proteínas totais, triglicérides (TG), colesterol associado à lipoproteína de alta (HDLc) e baixa (LDLC) densidade e glicemia de jejum (GJ). Nos períodos pós-operatórios (6, 12, 18, 24 meses) a avaliação nutricional foi feita pelas medidas de peso, perda ponderal, percentual de perda de peso (\%PP), IMC e bioquímica incluindo ferro, ferritina e transferrina. Resultados: $71,2 \%$ eram do sexo feminino, idade de 38,4 $\pm 9,96$ anos, $129,66 \pm 27,40 \mathrm{Kg}$ e IMC $48,6 \pm 8,9 \mathrm{Kg} / \mathrm{m}^{2}$, no préoperatório. Receberam o diagnóstico de SM 26,8\%, HA 52,7\% e DM 2 11,7\%. A bioquímica revelou TG, LDLc, GJ elevados, estando normais os demais parâmetros. Evolução antropométrica demonstrou perda ponderal progressiva, atingindo aos 24 meses IMC $31,7 \pm 5,82 \mathrm{Kg} / \mathrm{m}^{2}(\mathrm{p}<0,001)$ e maior \%PP $(36,05 \%)$. Valores de TG, LDLC e GJ atingiram a normalidade a partir do $6^{\circ}$ mês pósoperatório: $104,4 \mathrm{mg} / \mathrm{dL}(\mathrm{p}=0,018), 95,5 \mathrm{mg} / \mathrm{dL}(\mathrm{p}=0,263)$ e $84,8 \mathrm{~g} / \mathrm{dL}(\mathrm{p}=0,004)$, respectivamente; transferrina apresentou valores reduzidos aos 6 meses. Prevalência maior dos sintomas ocorreu no $6^{\circ}$ mês: alopécia (19\%), vômitos (18\%), intolerância alimentar (12,2\%). Conclusão: A Cirurgia bariátrica foi um procedimento eficaz para promover perda ponderal e sua manutenção por dois anos, assim como melhora de parâmetros bioquímicos e co-morbidades, com sintomas clínico-nutricionais reduzidos e/ou evitados por monitorização nutricional.
\end{abstract}

Descritores: Cirurgia bariátrica. Obesidade/metabolismo. Obesidade mórbida. Transtornos da alimentação. Síndrome metabólica. Nutrição. Cuidados pré-operatórios. Cuidados pós-operatórios.

\section{INTRODUÇÃO}

A obesidade mórbida, um estado de insulino-resistência por excelência ${ }^{1,2}$ está freqüentemente associada a síndrome metabólica (SM), condição que aumenta a mortalidade geral em cerca de 1,5 vez e a cardiovascular em aproximadamente 2,5 vezes ve $^{3,4}$. A prevalência de SM em obesos mórbidos, na maioria dos estudos, encontra-se acima de $50 \%$.

Existem evidências de que perdas de peso moderadas (5-10\% do peso inicial) com tratamentos convencionais, através de abordagens nutricionais, farmacológicas e atividade física promovem benéficos metabólicos ${ }^{6}$. Atualmente, porém, a ferramenta mais eficaz no tratamento e controle da obesidade mórbida é a intervenção cirúrgica7. Apesar de ser invasiva, tem alcançado resultados satisfatórios, com redução superior a $50 \%$ do excesso de peso ou 30 a $40 \%$ do peso inicial ${ }^{8}$. Estes benefícios são mantidos em longo prazo, determinando melhora nos parâmetros metabólicos ${ }^{9,10}$, com efeito positivo no metabolismo dos lipídeos e carboidratos, diminuindo a resistência à insulina e em muitos casos, contribuindo para o controle do diabetes tipo 2 (DM 2) e hiperlipidemias ${ }^{11,12}$.
Investigações têm demonstrado alterações no estado nutricional de pacientes submetidos à cirurgia bariátrica, as quais podem aparecer após semanas e/ou $\operatorname{anos}^{13}$. Os distúrbios nutricionais vão desde carências vitamínico-minerais (ferro, zinco, tiamina, niacina, ácido fólico, cobalamina, vitaminas $A, D$ e E) até manifestações de desnutrição energético-protéica ${ }^{14,15}$. Os possíveis mecanismos são: ingestão nutricional deficiente, má absorção decorrente da técnica cirúrgica, pobre aderência à reposição de polivitamínicos e a presença de sintomas gastrintestinais $^{16}$.

Considerando as repercussões da cirurgia bariátrica em obesos mórbidos, o objetivo deste estudo foi avaliar as características clínico-nutricionais desses pacientes, no pré e pós-operatório, assim como a presença de intolerâncias alimentares e sintomas comuns neste contexto.

\section{MÉTODOS}

O estudo caracterizou-se como sendo do tipo retrospectivo realizado no ambulatório de Nutrição/Cirurgia

Trabalho realizado no Hospital das Clínicas / Universidade Federal de Pernambuco - PE-BR.

1. Especialista em Nutrição pela Universidade Federal de Pernambuco - PE-BR; 2. Doutora em Nutrição pela Universidade Federal de Pernambuco - PE-BR; 3. Nutricionista graduada pela Universidade Federal de Pernambuco - PE-BR; 4. Nutricionista Residente pela Universidade Federal de Pernambuco - PE-BR. 
Geral do HC/UFPE. Foram avaliados 205 pacientes de ambos os sexos submetidos à cirurgia bariátrica, pela técnica Fobi-Capela (Bypass Gástrico em Y de Roux), durante o período de 2002 a 2006.

A análise considerou a investigação de história clínica para DM 2, hipertensão arterial (HA) e SM. A caracterização de SM considerou cinco parâmetros: circunferência abdominal (CA), pressão arterial e concentrações séricas de glicemia em jejum (GJ), triglicérides (TG) e de fração de colesterol de lipoproteína de alta densidade (HDLC), conforme proposto pelo NCEP-ATPIII ${ }^{17}$, em 2001, o qual preconiza o diagnóstico de SM na presença de pelo menos três dos seguintes critérios: $C A>88 \mathrm{~cm}$ para mulheres ou $>102 \mathrm{~cm}$ para homens, $\mathrm{HDLC}<50 \mathrm{mg} / \mathrm{dL}$ para mulheres ou $<40 \mathrm{mg} / \mathrm{dL}$ para homens, TG $>150 \mathrm{mg} / \mathrm{dL}$, pressão arterial com valores de corte considerando 130/ $85 \mathrm{mmHg}$ e GJ > $110 \mathrm{mg} / \mathrm{dL}$.

O estado nutricional pré-operatório foi avaliado pelo Índice de Massa Corporal (IMC) e dosagens bioquímicas: hemoglobina $(\mathrm{Hb})$, hematócrito $(\mathrm{Ht})$, albumina, proteínas totais, TG, HDLc e LDLc e GJ, realizados no HC/UFPE. Enquanto que nos períodos distintos de pós-operatório (6, 12, 18, 24 meses) a avaliação nutricional foi realizada por meio das medidas de peso, perda ponderal, percentual de perda de peso (\%PP), IMC e bioquímica, incluindo ferro, ferritina e transferrina. A classificação da obesidade foi realizada com adoção dos critérios da Organização Mundial de Saúde (OMS) de $1997^{18}$.

Para identificar a presença sintomas clíniconutricionais como intolerância alimentar, náuseas/vômitos, diarréia, gases, constipação, síndrome de dumping e alopécia foram coletados dados das fichas de atendimento nos distintos períodos.

Os dados foram analisados pelo software estatístico SPSS (Statistical Package for the Social Sciences) na versão $13^{19}$, com nível de significância estabelecido em $5,0 \%$, sendo utilizado teste t-Student pareado e obtidas distribuições absolutas e percentuais, sendo os resultados apresentados como: média, mediana, desvio padrão e coeficiente de variação.

Esta pesquisa foi previamente avaliada e aprovada pelo Comitê de Ética em Pesquisa em Seres Humanos do Centro de Ciências da Saúde da Universidade Fede- ral de Pernambuco (CEP/CCS/UFPE), de acordo com a Resolução N 196/96 do Conselho Nacional de Saúde, sob o registro $n^{\circ} 194 / 07$.

\section{RESULTADOS}

Foram avaliados 146 pacientes do sexo feminino $(71,2 \%)$ e 59 do masculino (28,8\%), com idade média de $38,4 \pm 9,6$ anos (20-59), sendo a faixa etária prevalente $=$ 31 anos e estado nutricional compatível com obesidade grau III (Tabela1).

Evidenciou-se SM em 26,8\% no pré-operatório. Neste mesmo período, foram diagnosticados DM 2 e HA em $11,7 \%$ e $52,7 \%$, respectivamente. No $6^{\circ}$ mês pós-operatório, apenas $2 \%$ permaneceram com HA, ocorrendo remissão em $100 \%$ dos casos de DM 2 .

A avaliação dos períodos pós-operatórios demonstrou redução gradativa do peso e IMC, com diferença estatística em todas as fases, atingindo com 18-24 meses o menor peso e IMC e maior \%PP (Tabela 2).

Com relação à bioquímica pré-operatória - $\mathrm{Hb}$, Ht, albumina, proteínas totais, HDLc - encontrava-se entre os valores referenciais, níveis de TG, LDLc e GJ mostraramse elevados, apresentando aos seis, 12 e 18 meses redução significativa, atingindo valores médios dentro da normalidade. A transferrina apresentou níveis reduzidos aos seis e 24 meses (Tabela 3).

Quanto aos sintomas clínico-nutricionais, observou-se maior prevalência no $6^{\circ}$ mês, com alopécia (19\%) e vômitos (18\%) (Figura 1); ao mesmo tempo a intolerância alimentar destacou-se neste período, apresentando variabilidade no pós-operatório analisado (Figura 2).

Algumas variáveis não puderam ser comparadas estatisticamente entre os quatro períodos de pós-operatório, devido ao reduzido percentual de retorno: $6(61,5 \%)$, $12(48,3 \%), 18(31,2 \%)$ e $24(24,9 \%)$ meses.

\section{DISCUSSÃO}

Segundo dados da OMS, o aumento da incidência da obesidade está ocorrendo em ambos os sexos e in-

Tabela 1 - Classificação de pacientes submetidos à cirurgia bariátrica, de acordo com o IMC.

\begin{tabular}{|c|c|c|c|c|c|c|c|c|c|c|}
\hline \multirow[t]{2}{*}{ Variável } & \multicolumn{2}{|c|}{ Pré-operatório } & \multicolumn{2}{|c|}{6 meses } & \multicolumn{2}{|c|}{12 meses } & \multicolumn{2}{|c|}{18 meses } & \multicolumn{2}{|c|}{24 meses } \\
\hline & $n$ & $\%$ & $\mathrm{n}$ & $\%$ & $\mathrm{n}$ & $\%$ & $n$ & $\%$ & $\mathrm{n}$ & $\%$ \\
\hline \multicolumn{11}{|l|}{$\cdot$ IMC } \\
\hline Eutrófico & - & - & 3 & 1,5 & 8 & 3,9 & 9 & 4,4 & 6 & 2,9 \\
\hline Pré-obeso & - & - & 18 & 8,8 & 34 & 16,6 & 20 & 9,8 & 19 & 9,3 \\
\hline Obesidade grau I & 2 & 1,0 & 39 & 19,0 & 38 & 18,5 & 29 & 14,1 & 20 & 9,8 \\
\hline Obesidade grau ॥ & 21 & 10,2 & 30 & 14,6 & 23 & 11,2 & 9 & 4,4 & 8 & 3,9 \\
\hline Obesidade grau III & 180 & 87,8 & 51 & 24,9 & 13 & 6,3 & 8 & 3,9 & 5 & 2,4 \\
\hline TOTAL & 203 & 99,0 & 141 & 68,8 & 116 & 56,6 & 75 & 36,6 & 58 & 28,3 \\
\hline
\end{tabular}


Tabela 2 - Avaliação antropométrica no pré-operatório e em períodos distintos de tempo do pós-operatório.

\begin{tabular}{|c|c|c|c|c|c|c|c|}
\hline Variável & Estatística & Pré-operatório & 6 meses & 12 meses & 18 meses & 24 meses & Valor de $p$ \\
\hline \multirow[t]{4}{*}{ Peso $(\mathrm{Kg})$} & Média & 129,66 & 99,88 & 86,85 & 83,88 & 83,94 & $\mathrm{p}^{(1)}<0,001 *$ \\
\hline & Mediana & 123,15 & 96,00 & 84,30 & 80,90 & 81,90 & $\mathrm{p}^{(I I)}<0,001 *$ \\
\hline & $\mathrm{DP}$ & 27,40 & 21,98 & 17,94 & 18,10 & 17,31 & $\mathrm{p}^{(I I I)}<0,001 *$ \\
\hline & CV & 21,13 & 22,01 & 20,66 & 21,58 & 20,62 & $\mathrm{p}^{(\mathrm{IV})}<0,001^{*}$ \\
\hline \multirow[t]{4}{*}{ Perda de peso $(\mathrm{Kg})$} & Média & & 30,35 & 43,09 & 47,15 & 47,49 & \\
\hline & Mediana & & 29,85 & 42,40 & 44,90 & 46,00 & \\
\hline & $\mathrm{DP}$ & & 9,52 & 11,66 & 13,98 & 13,62 & \\
\hline & CV & & 31,37 & 27,06 & 29,65 & 28,68 & \\
\hline \multirow[t]{4}{*}{$\%$ perda de peso } & Média & & 23,44 & 33,58 & 36,10 & 36,05 & \\
\hline & Mediana & & 24,15 & 33,75 & 35,50 & 36,10 & \\
\hline & $\mathrm{DP}$ & & 6,07 & 6,74 & 7,93 & 6,39 & \\
\hline & CV & & 25,90 & 20,07 & 21,97 & 17,72 & \\
\hline \multirow[t]{4}{*}{ IMC $\left(\mathrm{Kg} / \mathrm{m}^{2}\right)$} & Média & 48,63 & 36,98 & 32,59 & 31,71 & 31,38 & $\mathrm{p}^{(1)}<0,001 *$ \\
\hline & Mediana & 47,20 & 35,95 & 32,40 & 31,30 & 31,20 & $\mathrm{p}^{(I I)}<0,001$ * \\
\hline & $\mathrm{DP}$ & 8,98 & 7,80 & 6,04 & 5,82 & 5,50 & $\mathrm{p}^{(I I I)}<0,001 *$ \\
\hline & CV & 18,47 & 21,09 & 18,53 & 18,35 & 17,53 & $\mathrm{p}^{(\mathrm{IV})}<0,001$ * \\
\hline Circunferência & Média & 122,67 & 99,79 & 98,11 & 85,98 & & $\mathrm{p}^{(1)}<0,001 *$ \\
\hline \multirow[t]{3}{*}{ Abdominal $(\mathrm{cm})$} & Mediana & 122,00 & 101,75 & 95,00 & 81,50 & & $\mathrm{p}^{(I I)}<0,001$ * \\
\hline & $\mathrm{DP}$ & 14,96 & 18,07 & 14,77 & 13,98 & $\star *$ & $\star \star *$ \\
\hline & $C V$ & 12,20 & 18,11 & 15,05 & 16,26 & & $* * *$ \\
\hline
\end{tabular}

(**): Não foi determinado devido à informação está presente em um único paciente.

(I): Para comparação entre pré-operatório e 6 meses - teste t-Student pareado.

(II): Para comparação entre pré-operatório e 12 meses - teste t-Student pareado.

(III): Para comparação entre pré-operatório e 18 meses - teste t-Student pareado.

(IV): Para comparação entre pré-operatório e 24 meses - teste $t$-Student pareado.

dependente da classe social e nível cultural20. Porém, com maior prevalência do sexo feminino como foi encontrado em nosso serviço. Rangel et $a^{20}$ e Lehmann et $a^{R^{1}}$, reforçam esses dados com 76,6\% e $75 \%$ de mulheres, respectivamente, na amostra estudada. Em relação a faixa etária Quadros et a ${ }^{22}$ relataram média de 40 anos, semelhante a deste estudo e a descrita por Rangel et aRo.

Quanto à presença da SM, Lee et al. ${ }^{5}$ ao analisarem 645 obesos mórbidos, firmando o diagnóstico nos critérios do NCEP-ATP III, evidenciaram-na em 52,2\%; dados superiores aos da população estudada.

Similarmente ao que se observa na literatura, a prevalência de co-morbidades foi elevada. Rangel et $a^{20}$, relataram percentuais de $\mathrm{HA}$ em $60 \%$, seguida do DM 2 em 10\% dos pacientes. Contudo, Carvalho et $a^{R 3}$ e Santos et $a^{R^{4}}$ revelaram valores superiores em DM $231,9 \%$ e 35\%, respectivamente. Um aspecto que merece destaque é a considerável melhora das condições dos pacientes operados, em relação à HA $(79,4 \%)$ e ao DM 2 (100\%) em período inferior a seis meses ${ }^{25}$. Do mesmo modo que ocorreu com os pacientes desse serviço. Durante os 12 meses de pós-operatório, pela análise de Carvalho et a ${ }^{23}$, a grande maioria dos pacientes pode descontinuar o uso de anti-hipertensivos e todos apresentavam níveis normais de GJ. Confirmando esses achados, Valezi et $a^{26}$ analisando consecutivamente 250 pacientes, com seguimento de no míni- mo um ano, relataram a cura em $72,7 \%(32)$ do DM 2 e 65,4\%(34) de HA.

A melhora no metabolismo glicídio pode ser explicada pela severa privação de nutrientes que ocorre após a cirurgia, proporcionando perda de peso e balanço energético negativo, condições que classicamente meIhoram a tolerância à glicose ${ }^{23}$. Alterações de hormônios gastro-intestinais, como redução nos níveis plasmáticos de grelina e elevação de GLP1, é um outro mecanismo que pode agir em associação com o anterior ${ }^{27}$. A redução da hiperinsulinemia, da resistência à insulina, dos níveis de leptina ${ }^{27}$ e da pressão intra-abdominal estão relacionados com a melhora e/ou redução da pressão $\operatorname{arterial}^{28}$.

Na análise de perda de peso, identificou-se redução compatível com dados da literatura. Numa avaliação de 250 casos foi verificada uma redução de peso da ordem de $37,5 \%$ após o primeiro ano de cirurgia ${ }^{26}$. Por outro lado, Quadros et $a^{22}$, encontraram percentuais inferiores neste período (30,69\%). Vários pesquisadores constataram que a redução do peso é maior nos primeiros seis meses, passando a ser mais lenta e contínua22, até atingir perdas médias de 35 a $40 \%$ do peso inicial entre o $12^{\circ} \mathrm{e}$ $24^{\circ}$ mês da realização da cirurgia ${ }^{29}$. Com relação à redução no IMC estes dados são semelhantes aos encontrados por vários pesquisadores, onde Carvalho et a ${ }^{23}$, relataram

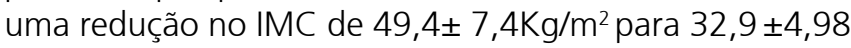


Tabela 3 - Avaliação bioquímica no pré-operatório e em períodos distintos de tempo do pós-operatório.

\begin{tabular}{|c|c|c|c|c|c|c|c|}
\hline Variável & Estatística & Pré-operatório & 6 meses & 12 meses & 18 meses & 24 meses & 5 Valor de $p$ \\
\hline \multirow[t]{4}{*}{ Hemoglobina ( $g / d L)$} & Média & 13,69 & 12,97 & 12,88 & 12,89 & 14,27 & $p^{(1)}=0,003 *$ \\
\hline & Mediana & 13,70 & 12,90 & 12,90 & 12,60 & 12,50 & $\mathrm{p}^{(I I)}=0,027^{*}$ \\
\hline & $\mathrm{DP}$ & 1,26 & 1,25 & 1,36 & 1,52 & 7,63 & $\mathrm{p}^{(I I I)}=0,003^{*}$ \\
\hline & CV & 9,20 & 9,64 & 10,56 & 11,79 & 53,47 & $p^{(I V)}=0,705$ \\
\hline \multirow[t]{4}{*}{ Hematocrito (\%) } & Média & 43,66 & 39,32 & 38,72 & 38,04 & 35,73 & $p^{(1)}=0,005^{*}$ \\
\hline & Mediana & 41,00 & 39,20 & 39,10 & 38,30 & 37,30 & $\mathrm{p}^{(I I)}=0,025^{*}$ \\
\hline & $\mathrm{DP}$ & 27,34 & 3,59 & 3,98 & 3,77 & 7,86 & $\mathrm{p}^{(I I I)}=0,003^{*}$ \\
\hline & CV & 62,62 & 9,13 & 10,28 & 9,91 & 22,00 & $\mathrm{p}^{(\mathrm{IV})}=0,049^{*}$ \\
\hline \multirow[t]{4}{*}{ Albumina $(\mathrm{g} / \mathrm{dL})$} & Média & 3,89 & 4,01 & 3,95 & 4,11 & 4,11 & $p^{(1)}=0,946$ \\
\hline & Mediana & 3,80 & 4,15 & 3,85 & 4,05 & 3,90 & $p^{(I I)}=0,618$ \\
\hline & $\mathrm{DP}$ & 0,44 & 0,64 & 0,50 & 0,64 & 0,58 & $\mathrm{p}^{(I I I)}=0,508$ \\
\hline & CV & 11,31 & 15,96 & 12,66 & 15,57 & 14,11 & $\mathrm{p}^{(\mathrm{IV})}=0,854$ \\
\hline \multirow[t]{4}{*}{ Transferrina (mg/dL) } & Média & - & 120,70 & 170,40 & 243,50 & 78,02 & $\star \star *$ \\
\hline & Mediana & - & 144,00 & 170,40 & 243,50 & 35,35 & $* * *$ \\
\hline & $\mathrm{DP}$ & - & 108,54 & 109,46 & 21,92 & 111,67 & $* * *$ \\
\hline & CV & - & 89,92 & 64,24 & 9,00 & 143,13 & $* * *$ \\
\hline \multirow[t]{4}{*}{ Ferro $(\mu \mathrm{g} / \mathrm{dL})$} & Média & - & 75,70 & 73,67 & 104,95 & 93,07 & $\star * *$ \\
\hline & Mediana & - & 66,20 & 65,70 & 95,70 & 94,60 & $* * *$ \\
\hline & DP & - & 29,20 & 38,11 & 69,67 & 31,86 & $* * *$ \\
\hline & CV & - & 38,57 & 51,73 & 66,38 & 34,23 & $\star * *$ \\
\hline \multirow[t]{4}{*}{ Ferritina $(\mathrm{mg} / \mathrm{ml})$} & Média & - & 115,83 & 168,32 & 135,90 & & $\star * *$ \\
\hline & Mediana & - & 52,60 & 108,00 & 71,15 & & $* * *$ \\
\hline & $\mathrm{DP}$ & - & 116,01 & 164,88 & 146,83 & $\star *$ & $* * *$ \\
\hline & CV & - & 100,15 & 97,96 & 108,04 & & $\star * *$ \\
\hline Proteínas & Média & 7,23 & 6,96 & 7,18 & 6,84 & 6,81 & $\star \star *$ \\
\hline \multirow[t]{3}{*}{ Totais $(\mathrm{g} / \mathrm{dL})$} & Mediana & 7,30 & 6,95 & 7,45 & 7,00 & 6,60 & $* * *$ \\
\hline & $\mathrm{DP}$ & 0,64 & 0,51 & 0,79 & 0,31 & 0,52 & $* \star *$ \\
\hline & CV & 8,85 & 7,33 & 11,00 & 4,53 & 7,64 & $\star \star \star$ \\
\hline \multirow[t]{4}{*}{ Triglicerídes (mg/dL) } & Média & 172,02 & 104,48 & 96,47 & 90,74 & 91,57 & $\mathrm{p}^{(1)}=0,018^{*}$ \\
\hline & Mediana & 133,00 & 94,80 & 85,00 & 79,00 & 90,00 & $\mathrm{p}^{(I I)}=0,037^{*}$ \\
\hline & $\mathrm{DP}$ & 121,24 & 44,64 & 46,17 & 43,03 & 31,10 & $\mathrm{p}^{(I I I)}=0,035^{*}$ \\
\hline & CV & 70,48 & 42,73 & 47,86 & 47,42 & 33,96 & $\mathrm{p}^{(\mathrm{IV})}=0,092$ \\
\hline
\end{tabular}

(**): Não foi determinado devido à informação estar presente em um único paciente.

$\left.{ }^{* * *}\right)$ : Não foi possível determinar devido a ocorrência de freqüências muito baixas.

(I): Para comparação entre pré-operatório e 6 meses - teste t-Student pareado.

(II): Para comparação entre pré-operatório e 12 meses - teste t-Student pareado.

(III): Para comparação entre pré-operatório e 18 meses - teste t-Student pareado.

(IV): Para comparação entre pré-operatório e 24 meses - teste $t$-Student pareado.

$\mathrm{Kg} / \mathrm{m}^{2}$ em 1 ano, semelhante aos resultados de Santos et $a^{R^{4}}$, com IMC de 50,4 $\pm 7,9 \mathrm{Kg} / \mathrm{m}^{2}$ para $31,9 \pm 5,85 \mathrm{Kg} / \mathrm{m}^{2}$, no mesmo período. Observou-se neste trabalho, assim como nos de outros autores, que após um ano os pacientes apresentaram IMC de obesidade grau l; embora apresentando uma redução do peso significativa, a maioria não atingiu o peso ideal.

Com relação à $\mathrm{HT}, \mathrm{Hb}$ e albumina Santos et $a^{R^{4}}$ detectaram valores adequados no pré-operatório, similares aos achados nesta pesquisa. Quanto à bioquímica no pósoperatório, Farias et $a l^{10}$, observaram concentração de albumina normal após 8 meses de cirurgia; em contrapartida, Skroubis et $a^{\beta 0}$, avaliando 243 pacientes, demonstraram baixa incidência de hipoalbuminemia e 37,7\% dos pacientes com baixos níveis de ferritina após 2 anos de cirurgia bariátrica, diferente do que foi observado neste grupo, possivelmente subestimados pelo reduzido número de exames realizados e baixo percentual de retorno. Relatos sugerem a necessidade da monitorização da ferritina, mantendo níveis superiores a $40 \mathrm{mcg} / \mathrm{dL}^{31}$. Dados da literatura referentes à bioquímica da nutrição em períodos de pós-operatórios semelhante ao estudado, são escassos, dificultando as comparações dos resultados.

A prevalência de dislipidemia é muito variável entre pacientes com obesidade mórbida, oscilando de 19 a $82,9 \%{ }^{32}$. Silva et $a^{\beta 3}$ e Carvalho et $a^{23}$ constataram elevação de TG e LDLc, com HDLc apresentando valores normais no pré-operatório, atingindo normalização de todos os parâmetros ao final de 12 meses. Achados que corroboram com os deste estudo. 


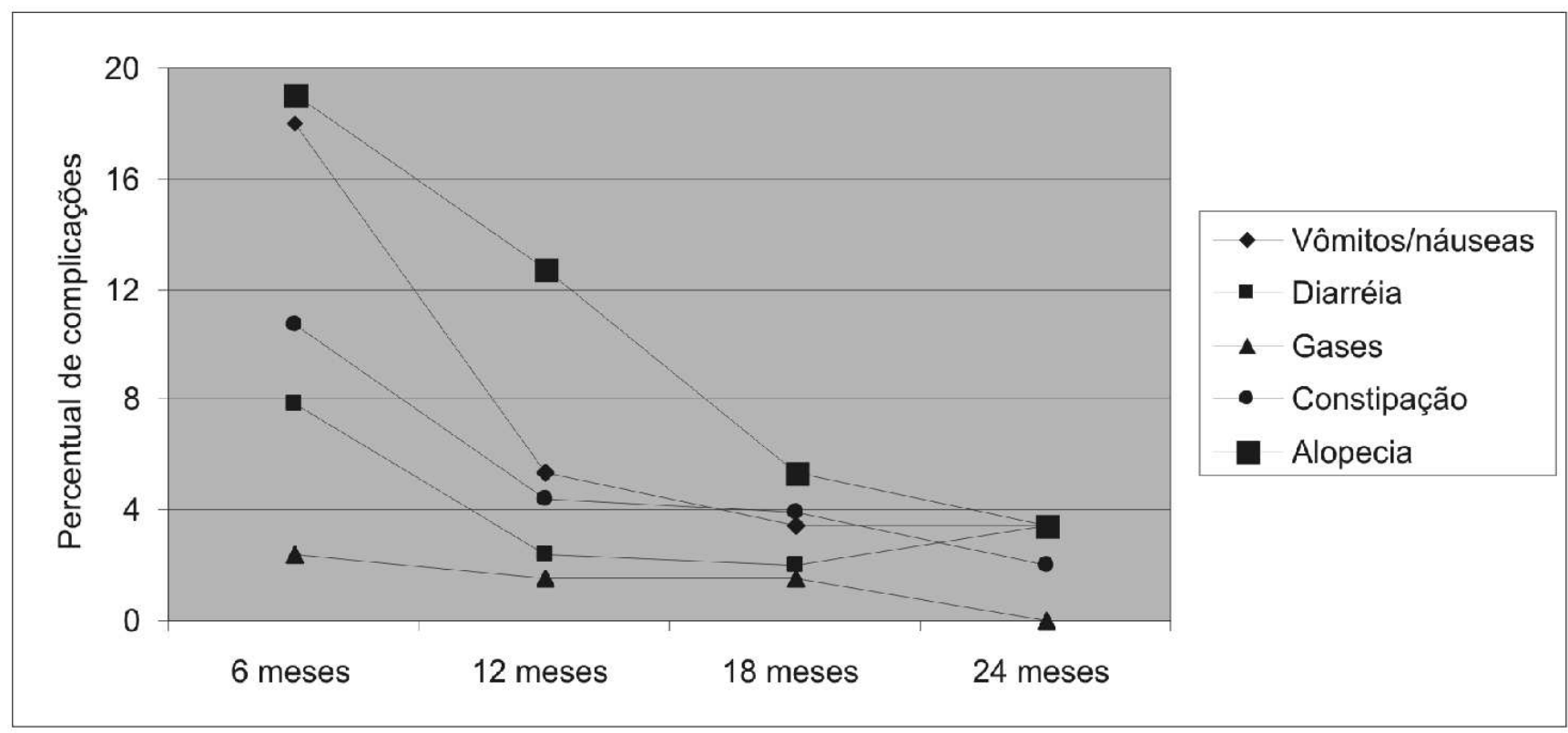

Figura 1 - Prevalência de sintomas clínico-nutricionais no pós-operatório.

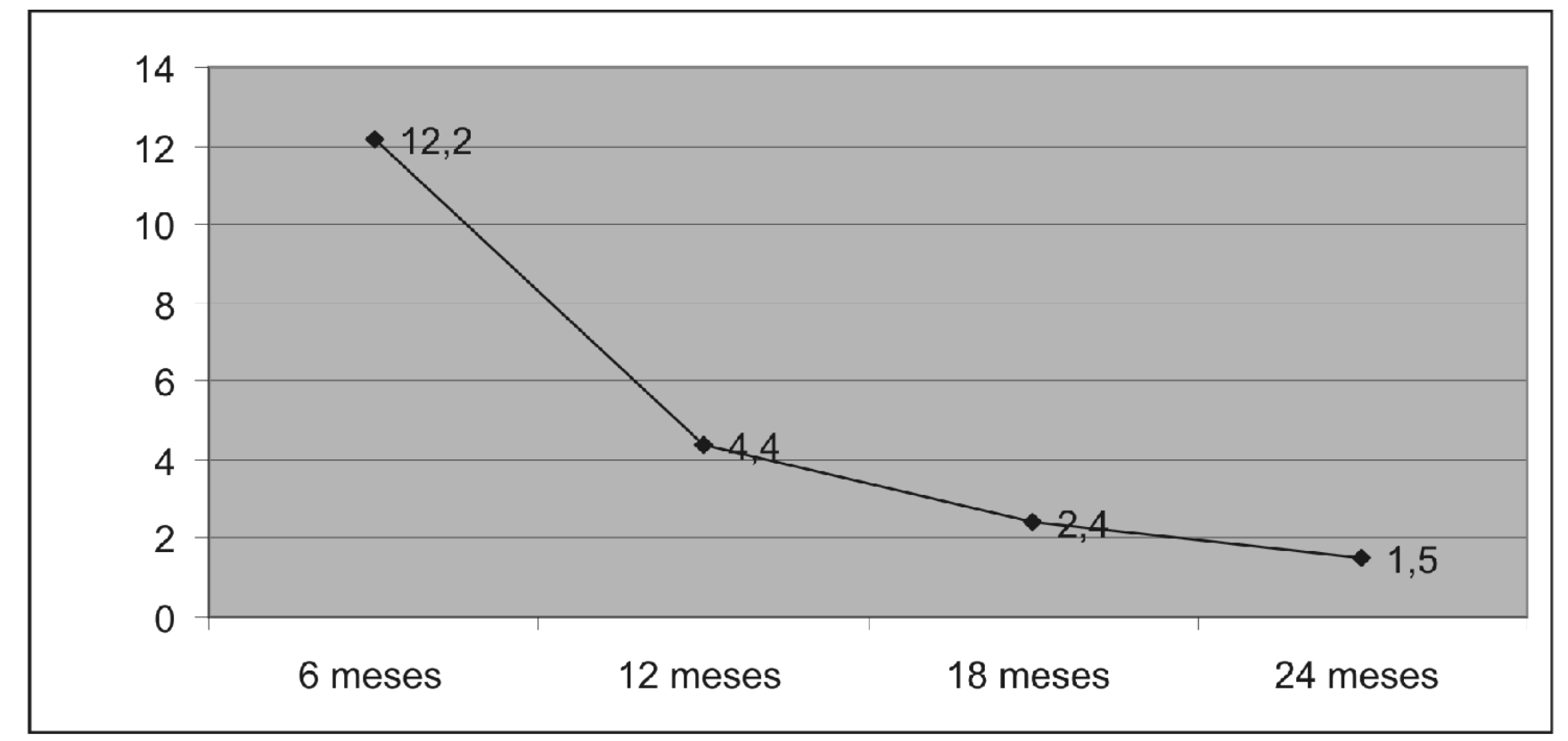

Figura 2 - Prevalência em percentuais de intolerância alimentar nos períodos distintos de pós-operatório.

Segundo Quadros et a ${ }^{R 2}$, vômitos (10,5\%), síndrome de dumping (7,01\%), diarréia (1,75\%) e constipação $(1,75 \%)$ foram pouco freqüentes nos primeiros seis meses de cirurgia, valores inferiores aos da presente pesquisa, com exceção da síndrome de dumping que não foi evidenciada nesse grupo, possivelmente pela exclusão na dieta de carboidrato simples por um mês, conduta estabelecida no protocolo do serviço. Por outro lado, Dias et $a^{\beta 4}$, observaram predominância de náusea (5\%) e alopécia (12\%), similares aos detectados nesta amostra, com exceção dos vômitos que foram superiores (45\%). A intolerância alimentar mostrou-se constante no pós-operatório (46,67\%) no estudo de Quadros et a/22, diferentemente deste trabalho, onde os percentuais mostraram-se reduzidos e decrescentes em cada período, provavelmente pela adesão às orientações nutricionais realizadas.

Antonini et $a^{\beta 5}$ e Cruz et $a^{\beta 6}$, referem que somente o acompanhamento nutricional adequado garante o sucesso da cirurgia, evitando complicações como vômitos, intolerância alimentar e perda de peso insuficiente, 
salientando-se a necessidade de atenção constante quanto ao fracionamento das refeições, mastigação e quantidade de alimentos ingeridos numa mesma refeição.

Estes resultados confirmam que a cirurgia bariátrica foi um procedimento eficaz para promover perda ponderal e sua manutenção por dois anos, sem a ocorrência de baixo peso, assim como melhora de parâmetros bioquímicos e co-morbidades, com sintomas clíniconutricionais reduzidos e/ou evitados por monitorização nutricional especializada.

\title{
A B S T R A C T
}

\begin{abstract}
Objective: To determine the physical-nutritional profile of obese patients submitted to bariatric surgery at the HC/UFPE. Methods: Two-hundred-and-five patients were evaluated retrospectively during the period of 2002 through 2006. Analysis considered clinical history for diabetes type 2 (DM 2), high blood pressure (HBP) and metabolic syndrome (MS). The preoperative nutritional status was evaluated by $\mathrm{MBI}$ and the biochemistry (hemoglobin, hematocrit, albumin, total proteins, triglycerides (TG), cholesterol associated with the lipoprotein of high (HDLC) and low (LDLC) density and fasting glycemia (FG). During the postoperative periods $(6,12,18,24$ months), we evaluated the nutritional status through measures of weight, weight loss, weight loss percentage (\%WL), MBI and biochemistry including iron, ferritin, transferrin. Results: Seventy-one and two-tenth percent were female, age was $38.4 \pm 9.96$ years, and MBI preoperative was $48.6 \pm 8.9 \mathrm{Kg} / \mathrm{m} 2$. MS diagnosis was present in $26.8 \%$, HBP was present in $52.7 \%$ and DM 2 was detected in $11.7 \%$. The biochemistry disclosed TG, it raised $L D L c$, and $F G$, and all other parameters were normal. The anthropometrical evolution demonstrated gradual loss, reaching at the 24 months, MBI $31.7 \pm 5.82 \mathrm{Kg} / \mathrm{m} 2(p<0.001)$ and greater $\% W L$, $36 \%$. Values of TG, LDLC and FG reached normality at the $6^{\text {th }}$ postoperative month: $104.4 \mathrm{mg} / \mathrm{dL}(p=0.018), 95.5 \mathrm{mg} / \mathrm{dL}(p=0.263)$ and $84.8 \mathrm{~g} /$ $d L(p=0.004)$ respectively; the transferrin showed reduced values at the $6^{\text {th }}$ month. A larger prevalence of the symptoms occurred in $6^{\text {th }}$ month: hair loss (19\%), vomiting (18\%), and food intolerances (12.2\%). Conclusion: The bariatric surgery was an efficient procedure to promote weight loss and its maintenance in two years, as well as improvement of biochemical parameters and comorbidities, with reduced clinical-nutritional symptoms and/or prevented by nutritional monitoring.
\end{abstract}

Key words: Bariatric surgery. Obesity/metabolism. Obesity, morbid. Eating disorders. Metabolic syndrome. Nutrition. Preoperative care. Postoperative care.

\section{REFERÊNCIAS}

1. Deitel M. Overweight and obesity worldwide now estimated to involve 1.7 billion people. Obes Surg. 2003; 13(3): 329-30.

2. Ferrannini E, Natali A, Bell P, Cavallo-Perin P, Lalic N, Mingrone G. Insulin resistance and hypersecretion in obesity. European Group for the Study of Insulin Resistance (EGIR). J Clin Invest. 1997; 100(5): 1116-73.

3. Lakka HM, Laaksonen DE, Lakka TA, Niskanen LK, Kumpusalo E, Tuomilehto J, Salonen JT. The metabolic syndrome and total and cardiovascular disease mortality in middle-aged men. JAMA. 2002; 288(21): 2709-16.

4. Girman CJ, Rhodes T, Mercuri M, Pyörälä K, Kjekshus J, Pedersen TR et al. The metabolic syndrome and risk of major coronary events in the Scandinavian Survival Study (4S) and the Air Force/Texas Coronary Atherosclerosis Prevention Study (AFCAPS/TexCAPS). Am J Cardiol. 2004; 93(2): 136-41.

5. Lee WJ, Huang MT, Wang W, Lin CM, Chen TC, Lai IR. Effects of obesity surgery on the metabolic syndrome. Arch Surg. 2004; 139(10): 1088-92

6. Case CC, Jones PH, Nelson K, O"Brian Smith E, Ballantyne CM. Impact of weight loss on the metabolic syndrome. Diabetes Obes Metab. 2002; 4(6): 407-14.

7. Soares CC, Falcão MC. Abordagem nutricional nos diferentes tipos de cirurgia bariátrica. Rev Bras Nutr Clin. 2007; 22(1): 59-64.

8. Choban PS, Jackson B, Poplawski S, Bistolarides P. Bariatric surgery for morbid obesity: why, who, when, how, where, and then what? Cleve Clin J Med. 2002; 69(11): 897-903.

9. Brolin RE. Bariatric surgery and long-term control of morbid obesity. JAMA. 2002; 288(22): 2793-6.

10. Farias LM, Coêlho MPSS, Barbosa RF, Santos GS, Marreiro DN. Aspectos nutricionais em mulheres obesas submetidas à gastroplastia vertical com derivação gastro-jejunal em Y-de- Roux. Rev Bras Nutr Clin. 2006; 21(2): 98-103.

11. Buchwald $H$, Avidor $Y$, Braunwald $E$, Jensen MD, Pories W, Fahrbach $\mathrm{K}$, Schoelles K. Bariatric surgery. A systematic review and meta- analysis. JAMA. 2004; 292(14): 1724-37. Erratum in: JAMA. 2005; 293(14): 1728.

12. Muscelli E, Mingrone G, Camastra S, Manco M, Pereira JA, Pareja $J C$, Ferrannini E. Differential effect of weight loss on insulin resistance in surgically treated obese patients. Am J Med. 2005; 118(1): 51-7.

13. Sociedade Brasileira de Cirurgia Bariátrica e Metabólica. Resolução CFM n० 1.766/05. Disponível em: http:// www.portalmedico.org.br/resolucoes/cfm/2005/ 1766_2005.htm

14. Nautiyal A, sing S, Alaimo DJ. Wernicke encefalophathy-- an emerging trend after bariatric surgery. Am J Med. 2004; 117(10): 804-5.

15. Bloomberg RD, Fleishman A, Nalle JE, Herron DM, Kini S. Nutritional deficiencies following bariatric surgery: what have we learned? Obes Surg. 2005; 15(2): 145-54.

16. Alves LFA, Gonçalves RM, Cordeiro GV, Lauria MW, Ramos AV. Beribéri pós bypass gástrico: uma complicação não tão rara. Relato de dois casos e revisão da literatura. Arq Bras Endocrinol Metab. 2006; 50(3): 564-68.

17. The Third Report of the National Cholesterol Education Program (NCEP). Expert Panel on Detection. Evaluation and treatment of high blood cholesterol in adults (Adult Treament Panel III). JAMA. 2001; 285(19): 2486-97.

18. World Health Organization - WHO. Obesity: prevention ad management of the global epidemic. The WHO consultation on obesity. Geneve 3-5, 1997.

19. Statistical Package for the Social Sciences (SPSS) for Windows (Software) versão 13. Chicago (IL): SPSS Inc, 2004.

20. Rangel LOB, Faria VSP, Magalhães EA, Araújo ACT, Bastos EMRD. Perfil de saúde e nutricional de pacientes portadores de obesidade mórbida candidatos à cirurgia bariátrica. Rev Bras Nutr Clin. 2007; 22(3): 214-9.

21. Lehmann ALF, Valezi AC, Brito EM, Marson AC, Souza JCL. Correlação entre hipomotilidade da vesícula biliar e desenvolvimento de colecistolitíase após operação bariátrica. Rev Col Bras Cir. 2006; 33(5): 285-8. 
22. Quadros MRR, Savaris AL, Ferreira MV, Branco Filho AJ. Intolerância alimentar no pós-operatório de pacientes submetidos à cirurgia bariátrica. Rev Bras Nutr Clin. 2007: 22(1): 15-9.

23. Carvalho PS, Moreira CLCB, Barelli MC, Oliveira FH, Guzzo MF, Miguel GP, Zandonade E. Cirurgia bariátrica cura síndrome metabólica? Arq Bras Endocrinol Metab. 2007; 51(1): 79-85.

24. Santos EMC, Burgos MGPA, Silva SA. Perda ponderal após cirurgia bariátrica de Fobi-Capella: realidade de um hospital universitário do nordeste brasileiro. Rev Bras Nutr Clin. 2006; 21(3): 188-92.

25. Faria OP, Pereira VA, Gangoni CMC, Lins RD, Leite S, Rassi V et al. Obesos mórbidos tratados com gastroplastia redutora com bypass gástrico em Y de roux: análise de 160 pacientes. Brasília Med. 2002; 39(1/4): 6-34.

26. Valezi AC, Júnior JM, Brito EM, Marson AC. Gastroplastia vertical com bandagem em Y-de-roux: análise de resultados. Rev Col Bras Cir. 2004; 31(1): 49-56.

27. Hojo NES, Melo JM, Nobre LN. Alterações hormonais após cirurgia bariátrica. Rev Bras Nutr Clin. 2007; 22(1): 77-82.

28. Geloneze B, Preja JC. Cirurgia bariátrica cura a síndrome metabólica? Arq Bras Endocrinol Metab. 2006; 50(2): 400-7.

29. Garrido Júnior AB, Ferraz EM, Barroso FL, et al. Cirurgia em obesidade. São Paulo: Atheneu; 2003.

30. Skroubis G, Sakellaropoulos G, Pouggouras K, Mead N, Nikiforidis $G$, Kalfarentzos F. Comparison of nutritional deficiencies after Rouxen-Y gastric bypass and after biliopancreatic diversion with Rouxen-Y gastric bypass. Obes Surg. 2002; 12(4): 551-8.

31. Kushner R. Managing the obese patient after bariatric surgery: a case report of severe malnutrition and review of the literature. JPEN J Parenter Enteral Nutr. 2000; 24(2): 126-32.
32. Ferraz EM, Arruda PCL, Bacelar TC, Ferraz AAB, Albuquerque AC, Leão CS. Tratamento cirúrgico da obesidade mórbida. Rev Col Bras Cir. 2003; 30(2): 98-105.

33. Silva EN, Sanches MD. Perfil lipídico de obesos antes e após a derivação gástrica a Fobi-Capela. Rev Col Bras Cir. 2006; 33(2): 91-5.

34. Dias MCG, Ribeiro AG, Scabin VM, Faintuch J, Zilberstein B, GamaRodrigues JJ. Dietary intake of female bariatric patients after antiobesity gastroplasty. Clinics (São Paulo). 2006; 61(2): 93-8. Epub 2006 Apr 25.

35. Antonini DR, Pereira CRV, Simões $N$ et al. Avaliação nutricional de pacientes submetidos à cirurgia bariátrica. Bol Cirur Obes 2001; 2(4): 3.

36. Cruz MRR, Morimoto IMI. Intervenção nutricional no tratamento cirúrgico da obesidade mórbida: resultados de um protocolo diferenciado. Rev Nutr. 2004; 17(2): 263-72.

Recebido em 10/11/2008

Aceito para publicação em 16/01/2009

Conflito de interesse: nenhum

Fonte de financiamento: nenhuma

\section{Como citar este artigo:}

Pedrosa IV, Burgos MGPA, Souza NC, Morais CN. Aspectos nutricionais em obesos antes e após a cirurgia bariátrica. Rev Col Bras Cir. [periódico na Internet] 2009; 36(4). Disponível em URL: http://www.scielo.br/rcbc

\section{Endereço para correspondência:}

Isabella Valois Pedrosa

E-mail: isabella_valois@hotmail.com 